\title{
Mixed Diffusion-Controlled Growth of Pearlite in Binary Steel
}

\author{
By A. S. Pandit And H. K. D. H. Bhadeshia \\ University of Cambridge \\ Materials Science and Metallurgy \\ Pembroke Street, Cambridge CB2 3QZ, U. K.
}

\begin{abstract}
A kinetic theory for the diffusion-controlled growth of pearlite is presented, which accounts simultaneously for diffusion through the austenite and via the transformation front. The simplified method abandons the need for mechanical equilibrium at the phase junctions and yet is able to explain experimental data on the growth rate of pearlite. Furthermore, unlike previous analyses, the deduced value for the activation energy for the interfacial diffusion of carbon is found to be realistic when compared with corresponding data for volume diffusion.
\end{abstract}

\section{Introduction}

A colony of pearlite when viewed in three dimensions consists of an interpenetrating bicrystal of ferrite and cementite (Graef et al., 2006; Hillert, 1962; Kral et al., 2000). In planar sections the phases appear as lamellae which grow at a common front with the austenite. Cementite $(\theta)$ is rich in carbon whereas ferrite $(\alpha)$ accommodates very little when it is in equilibrium with either cementite or austenite $(\gamma)$. It is therefore necessary for carbon to be redistributed at the transformation front. This can happen either by diffusion in the austenite in a direction parallel to the transformation front, or by the migration of solute atoms within the $\alpha / \gamma$ and $\theta / \gamma$ interfaces. When the mobility of the interface is large, both of these mechanisms are said to be diffusion-controlled, i.e., most of the available free energy is dissipated in driving diffusion, (Bhadeshia, 1985).

For a given interlamellar spacing, it has long been possible to estimate the growth rate of pearlite assuming volume diffusion-control (Hillert, 1957; Zener, 1946) and the work has been reviewed thoroughly in (Christian, 2003a,b; Hillert, 1970; Ridley, 1984). There have, on the other hand, been reports (Brown and Ridley, 1966; Frye et al., 1953; Pearson and Verhoeven, 1984) that the rates calculated in this way significantly underestimate those measured, possibly because of mass transport within the transformation interface (Hillert, 1972; Sundquist, 1968). Such a mechanism has also been considered in the context of cellular precipitation where the cell boundary provides an easy diffusion path, with an activation energy for the boundary diffusion coefficient which was less than half that for volume diffusion (Turnbull, 1955). 
There have been two further explanations proposed for the faster than expected growth rate of pearlite. The first is that transformation strain enhances diffusion (Pearson and Verhoeven, 1984), but this neglects the fact that pearlite forms by reconstructive transformation in which case transformation strains should not be significant; furthermore, it has not been necessary to invoke such an argument in the case of other reconstructive transformations where the closure between experiment and theory is satisfactory (Bhadeshia, 1985).

In recent work using phase-field modelling, it has been argued that the rate of growth is enhanced by the diffusion of carbon through the ferrite, leading to a thickening of cementite behind the transformation front (Nakajima et al., 2006). The evidence for such thickening in micrographs of partially transformed specimens is not strong (see for example, Fig. 4 of (Darken and Fisher, 1962)). It was also admitted by the authors that there are uncertainties in the phase field method introduced by adjustable parameters and compromises necessary to ensure numerical stability. Their analysis also neglected to consider the flux through the transformation front. It is worth noting that Nakajima et al. (2006) calculated growth rates which were significantly smaller than experimental data, and hence speculated a role of transformation strain in enhancing diffusion (Pearson and Verhoeven, 1984). We will show later that this is unnecessary when the role of the inevitable flux through the transformation front is taken into account.

This highlights a general problem, that most comparisons between experiment and theory have been based on assumptions of either volume or interface-diffusion control; in other words, mechanisms in isolation. Fluxes through both of these processes must in practice contribute to diffusion and the relative contributions from each of these mechanisms will vary with circumstances. An elegant and first attempt at dealing simultaneously with interface and volume diffusion in $\mathrm{Fe}-\mathrm{C}$ alloys is due to Hashiguchi and Kirkaldy (1984). For diffusion-controlled growth they assumed parallel mass transfer in the volume ahead of the interface and through the interface, allowing for the Gibbs-Thompson effect at both the $\gamma / \theta$ and $\gamma / \alpha$ boundaries, and for mechanical equilibrium at the various interfacial junctions. The result was a rather complex theory which could not be implemented without making important approximations:

- in spite of the requirement of mechanical equilibrium, the interfaces with austenite were approximated as being flat except in the close proximity of the three-phase junctions;

- the distribution coefficient describing the ratio of the composition in the austenite in contact with ferrite or cementite, and in the transformation front was assumed to be constant, even though the interfacial energies $\sigma^{\gamma \alpha}$ and $\sigma^{\gamma \theta}$ are not expected to be identical;

- a simplification was made that $\sigma^{\gamma \alpha} \propto \sigma^{\alpha \theta}$ and $\sigma^{\gamma \theta} \propto \sigma^{\alpha \theta}$.

Whilst these approximations are entirely understandable, some are clearly inconsistent with the detailed theory and the whole problem might be simplified by abandoning the need for mechanical equilibrium. Indeed, it is not strictly necessary during growth when the rate of free energy dissipation is large, for equilibrium configurations to be respected as long as the process leads to a net reduction in 
free energy. This can be seen during two-dimensional grain growth simulations assuming orientation-independent boundary energies, where the triple junctions do not maintain $120^{\circ}$ angles during the process of growth, as might be required by mechanical equilibrium (Suwa and Saito, 1997). Another analogy is phase transformation where the chemical potential of a particular solute can increase with the passage of the interface as long as the overall free energy is reduced.

When the approximated model (Hashiguchi and Kirkaldy, 1984) was fitted to experimental data, rather large $\sigma^{\alpha \theta}$ interfacial energies were obtained, and the activation energy for the boundary diffusion of carbon was deduced to be in the range of 159920-169925 $\mathrm{J} \mathrm{mol}^{-1}$, which surprisingly is greater than for volume diffusion in both ferrite and austenite. It is noteworthy that Sundquist (1968) reported an even larger activation energy for the boundary diffusion of carbon, commenting that the expected value should be much smaller; he attributed the discrepancy to a possible role of substitutional solute impurities.

The goal in the present work was to derive a simplified theory which still deals with diffusion simultaneously through both boundary and volume and to compare the data against experiments. It will be seen that a number of difficulties, including that associated with the activation energy described above, are resolved.

\section{Model Formulation}

(a) Assumptions

(i) To be consistent with diffusion-controlled growth, local equilibrium is assumed to exist at the interfaces so that the chemical potentials $\mu$ of all elements are uniform there:

$$
\mu_{F e}^{\gamma}=\mu_{\mathrm{Fe}}^{\alpha} \quad \text { and } \mu_{\mathrm{C}}^{\gamma}=\mu_{\mathrm{C}}^{\alpha}
$$

It follows that the compositions where the different phases are in contact are given by tie-lines of the equilibrium $\mathrm{Fe}-\mathrm{C}$ phase diagram, which was calculated using MTDATA (NPL, 2006) and the TCFE database, Fig. 1.

(ii) Since the kinetic theory gives the growth rate as a function of interlamellar spacing rather than a unique velocity, it is assumed that the actual spacing adopted is that which leads to a maximum in the rate of entropy production (Hashiguchi and Kirkaldy, 1984) although the maximum growth rate criterion (Zener, 1946) is also considered for the sake of completeness.

(iii) The model is created for conditions in which fluxes from diffusion within the austenite ahead of the transformation front, and that via transport through the transformation front both contribute to growth.

\section{(b) Diffusion Coefficients}

The diffusion coefficient $D_{V}$ of carbon in austenite is strongly dependent on concentration (Darken, 1949; Wells et al., 1950). A particular model which accounts for the thermodynamics and carbon-carbon interactions whilst representing the known data rather well is as follows (Bhadeshia, 1981; Siller and McLellan, 1970):

$$
D_{V}=\frac{k T}{h} \exp \left(\frac{-\Delta G^{a}}{k T}\right)\left(\frac{\lambda^{2}}{3 \Gamma_{m}}\right) \eta
$$

Proc. Royal Society A, 467 (2011) 508-521 


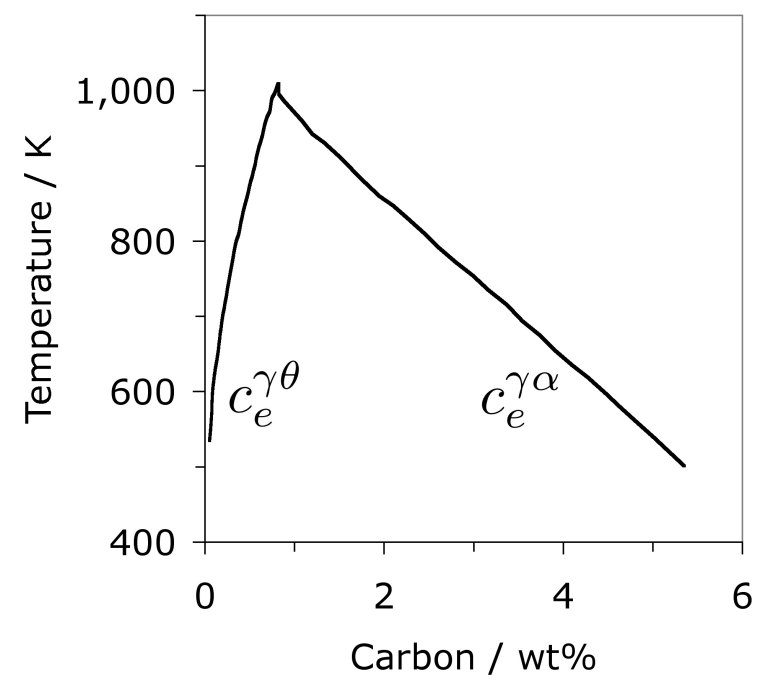

Figure 1. The extrapolated phase boundaries for equilibrium between austenite and cementite, and austenite and ferrite, in the $\mathrm{Fe}-0.8 \mathrm{C}$ wt\% system.

$k$ and $h$ are the Boltzmann and Planck constants respectively and

$$
\eta=a_{\gamma}\left[1+\frac{z(1+\chi)}{1-(0.5 z+1) \chi+\left[0.25 z^{2}+0.5 z\right][1-\phi] \chi^{2}}+(1+\chi) \frac{\partial a_{\gamma}}{\partial \chi}\right]
$$

where $\chi$ is the atom fraction of carbon, $z$ is the number of octahedral interstices around a single such interstice ( $z=12$ for austenite), $\Delta G^{a}$ is the activation free energy, $\Gamma_{m}$ is the activity coefficient of the activated complex, $\lambda$ is an interplanar spacing in the austenite, and $a_{\gamma}$ is the activity of carbon in austenite. The activity and its partial differential with respect to $\chi$ are determined using a quasichemical thermodynamic solution model (Bhadeshia, 1980; Dunn and McLellan, 1970). The term $\phi$ is given by

$$
\phi=1-\exp \left(\frac{-\omega_{\gamma}}{k T}\right)
$$

where $\omega_{\gamma}$ is the nearest neighbour carbon-carbon interaction energy of $8250 \mathrm{~J} \mathrm{~mol}^{-1}$ (Dunn and McLellan, 1970). Bhadeshia found $\Delta G^{a} / k=21230 \mathrm{~K}$ and $\ln \left\{\Gamma_{m} / \lambda^{2}\right\}=$ 31.84 (Bhadeshia, 1981).

Since the diffusion coefficient of carbon in austenite is strongly concentration dependent, and given that concentration gradients drive the flux, it is necessary to calculate a weighted average coefficient (Trivedi and Pound, 1967):

$$
\bar{D}_{V}=\int_{c_{e}^{\gamma \theta}}^{c_{e}^{\gamma \alpha}} \frac{D\left\{c^{\gamma}, T\right\}}{c_{e}^{\gamma \alpha}-c_{e}^{\gamma \theta}} d c^{\gamma}
$$

where $c_{e}^{\gamma \alpha}$ and $c_{e}^{\gamma \theta}$ are the mole fractions of carbon in austenite which is in equilibrium with ferrite and cementite respectively.

\section{(c) Combined Fluxes During Pearlite Growth}

A model is developed here which accounts for fluxes through both the austenite and within the transformation front, on average parallel to the front. For reasons 
described in the introduction, the model abandons the notion of interfacial tensions being balanced at three-phase junctions. As in previous work, it is assumed that diffusion within the interface can be described by a single coefficient, rather than two separate values corresponding to the $\alpha / \gamma$ and $\theta / \gamma$ interfaces. Fig. 2 illustrates the geometry of the pearlite colony.

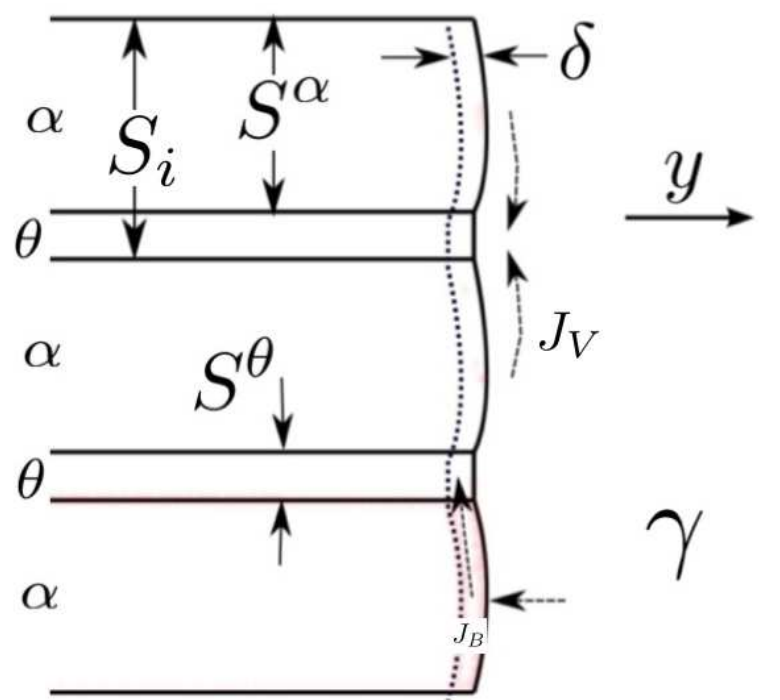

Figure 2. Geometry of pearlite colony. The dashed arrows indicate the volume and interface diffusion processes. The thickness of the boundary is written $\delta$.

The flux $J_{V}$ away from the ferrite (equal to that towards the cementite), through the volume of the austenite is given by:

$$
J_{V}=-\frac{A^{\alpha}}{V_{m}} \bar{D}_{V} \frac{d c}{d x}=\frac{\bar{D}_{V} b S^{\alpha}}{V_{m}} \frac{\left(c^{\gamma \alpha}-c^{\gamma \theta}\right)}{S^{\alpha} / 2}
$$

where $V_{m}$ is the molar volume of austenite $\left(7.1 \times 10^{-6} \mathrm{~m}^{3} \mathrm{~mol}^{-1}\right)$ and to a good approximation assumed to be the same for all the phases involved, and $A^{\alpha}$ is the cross sectional area of the interface, which for a unit depth into the diagram (Fig. 2) is equal to $S^{\alpha}$, and the diffusion distance parallel to the interface, from the ferrite to the cementite is on average $S^{\alpha} / 2$. An equation similar to the one above can be written for the boundary-diffusion flux $J_{B}$ of carbon through the interface between austenite and ferrite towards the cementite (Hillert, 1970):

$$
J_{B}=-\frac{A^{\alpha}}{V_{m}} D_{B} \frac{d c}{d x}=\frac{12 D_{B} \delta\left(c^{\gamma \alpha}-c^{\gamma \theta}\right)}{V_{m} S_{i}}
$$

Interfaces are created between cementite and ferrite during the growth of pearlite, thus consuming some of the free energy $\Delta G$ of transformation. All of the available free energy is consumed in this way when the spacing between lamellae reaches a critical value $S_{c}=2 \sigma V_{m} / \Delta G$, where $\sigma$ is the $\theta / \alpha$ interfacial energy per unit area. The growth rate then becomes zero but for $S_{i}>S_{c}$ the free energy change is

Proc. Royal Society A, 467 (2011) 508-521 
reduced by a factor $\left(1-S_{c} / S_{i}\right)$ and the concentration difference driving diffusion becomes $\left(c_{e}^{\gamma \alpha}-c_{e}^{\gamma \theta}\right)\left(1-S_{c} / S_{i}\right)$ (Zener, 1946).

The total flux arriving at the $\theta / \gamma$ interface is a combination from transport through the volume of austenite and via the boundary. It follows that for a growth velocity $v$, the material balance at the transformation front is given by:

$$
\frac{v S^{\alpha}}{V_{m}}\left(\bar{c}-c^{\alpha}\right)=\frac{v S^{\theta}}{V_{m}}\left(c^{\theta}-\bar{c}\right)=\frac{v S^{\alpha} S^{\theta}}{S_{i} V_{m}}\left(c^{\theta}-c^{\alpha}\right)
$$

Combining equations 2.5, 2.6 and 2.7 yields:

$$
\frac{v S^{\alpha} S^{\theta}}{S_{i}}\left(c^{\theta}-c^{\alpha}\right)=2 \bar{D}_{V}\left(c^{\gamma \alpha}-c^{\gamma \theta}\right)+\frac{12 D_{B} \delta\left(c^{\gamma \alpha}-c^{\gamma \theta}\right)}{S_{i}}
$$

where $\bar{c}$ is the average concentration in the austenite. The growth velocity is now isolated as follows:

$$
v=\left(\frac{c_{e}^{\gamma \alpha}-c_{e}^{\gamma \theta}}{c^{\theta}-c^{\alpha}}\right)\left(2 \bar{D}_{V}+\frac{12 D_{B} \delta}{S_{i}}\right) \frac{S_{i}}{S^{\alpha} S^{\theta}}\left(1-\frac{S_{c}}{S_{i}}\right)
$$

The problems associated with using a correct boundary diffusion coefficient have already been emphasised. It was decided therefore to deduce this using measured data on growth rate and interlamellar spacing, due to Brown and Ridley (1966), based on the more reliable method of size distributions rather than the observation of what might be the largest colony. Given that $D_{V}$ is well established, the only unknown then becomes $D_{B}$ (Fig. 3), from which an activation energy for interfacial diffusion during the pearlite reaction was derived to be $Q_{B} \approx 97 \mathrm{~kJ} \mathrm{~mol}^{-1}$, with

$$
D_{B}=8.51 \times 10^{-5} \exp \left(-\frac{96851}{R T}\right) \mathrm{m}^{2} \mathrm{~s}^{-1}
$$

It is interesting that unlike previous work (Hashiguchi and Kirkaldy, 1984; Offerman et al., 2003) where the activation energy for boundary diffusion was found to be greater than for volume diffusion in both austenite and ferrite, here $Q_{B}$ is bracketed between $Q_{V}^{\alpha}=70 \mathrm{~kJ} \mathrm{~mol}^{-1}$ and $Q_{V}^{\gamma}=135 \mathrm{~kJ} \mathrm{~mol}^{-1}$ (Honeycombe and Bhadeshia, 1995).

The ratio of boundary to volume diffusion flux is shown as a function of temperature in Fig. 4; as might be expected, boundary diffusion dominates except at the highest of transformation temperatures.

\section{Spacing Criteria}

There are two criteria common in fixing the interlamellar spacing of pearlite; one involves the maximisation of growth rate (Zener, 1946), and the other, of the entropy production rate (Kirkaldy and Sharma, 1980). For volume diffusion-controlled growth, $S / S_{c}$ is 2 and 3 , and in the case of interfacial diffusion controlled growth it is 1.5 and 2 for the maximum growth and entropy production criteria respectively. We now consider how these numbers are modified for the mixed volume and interface-diffusion modes. 

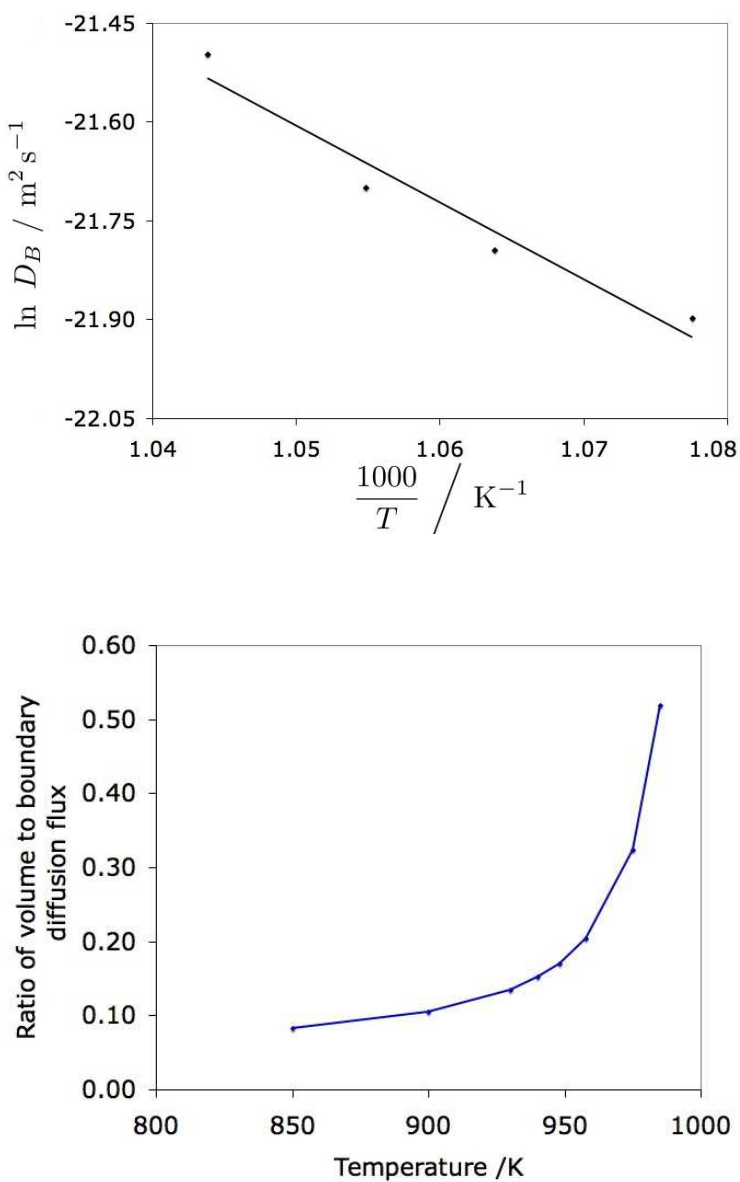

Figure 3. Arrhenius plot of $D_{B}$ versus inverse of temperature in $\mathrm{Fe}-0.8 \mathrm{C}$ wt\% steel for mixed mode diffusion controlled pearlite growth

Figure 4. Relative contributions of volume and boundary diffusion fluxes during the formation of pearlite in $\mathrm{Fe}-0.8 \mathrm{C}$ wt\% steel.

During isothermal transformation, the rate of entropy production is (Cahn and Hagel, 1962; Kirkaldy and Sharma, 1980)

$$
\dot{S}=\frac{v\left(\Delta G_{\text {net }}\right)}{T}
$$

where $\Delta G_{\text {net }}=\Delta G-2 \sigma / S_{i}$ is the free energy dissipated; $\Delta G$ is the total driving force for the austenite to pearlite transformation. If it is assumed that for small undercoolings below the eutectoid temperature $T_{E}$ the entropy change of transformation, $\Delta S$ is constant, then $\Delta G=\Delta H\left(1-T / T_{E}\right)$ where $\Delta H$ is the corresponding enthalpy change, then this equation becomes

$$
\dot{S}=v \frac{\Delta H \Delta T}{T_{E}}\left(1-\frac{S_{c}}{S_{i}}\right)
$$

Using equation 2.9 this becomes:

$$
\dot{S}=\beta\left(\frac{2 \bar{D}_{V} S_{i}+12 D_{B} \delta}{S_{i}^{2}}\right) \frac{\Delta H \Delta T}{T_{E}}\left(1-\frac{S_{c}}{S_{i}}\right)^{2}
$$

Proc. Royal Society A, 467 (2011) 508-521 
where $\beta$ contains the concentration terms. $\dot{S}$ is plotted as a function of $S_{i} / S_{c}$ in Fig. 5a; the maximum in $\dot{S}$ is obtained when $S_{i} / S_{c}$ is between 2.01 to 2.17 depending on the temperature of transformation, but independent of the interfacial energy $\sigma$. The corresponding calculations for the maximum growth rate criterion are shown in Fig. 5b; the values of $S_{i} / S_{c}$ vary from 1.36 to 1.53 as a function of temperature. It is not surprising that the values are similar to those expected for the case where only interface diffusion occurs, since this is the dominant process at all temperatures (Fig. 4).

(a)
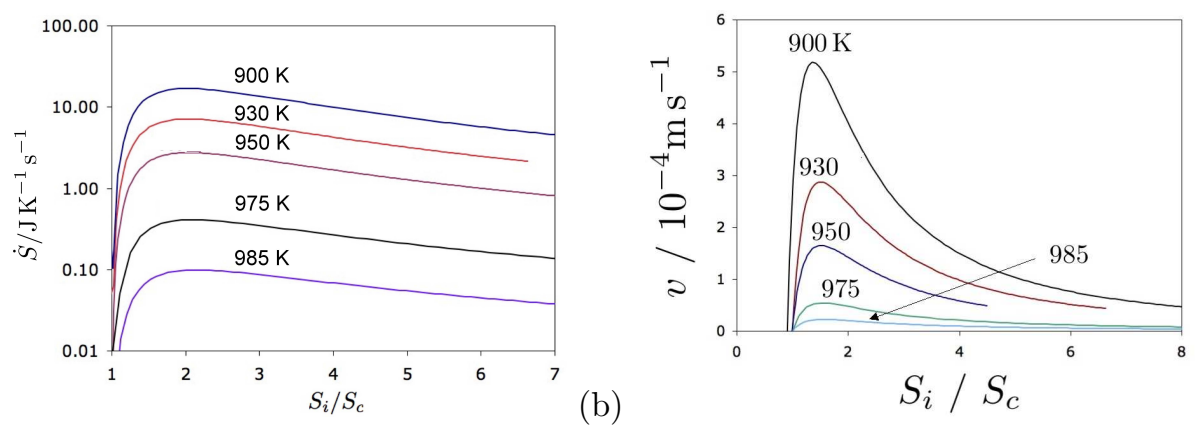

Figure 5. (a) Variation in the entropy production rate as a function of the normalised interlamellar spacing (b) Similar plot for the maximum growth rate.

\section{(a) Interfacial energy}

It is possible to derive from the kinetic data on pearlite growth, the interfacial energy $\sigma$ relating cementite and ferrite (Hashiguchi and Kirkaldy, 1984)

$$
\sigma=\frac{1}{2} S_{c} \Delta G \approx \frac{S_{c} \Delta T \Delta H}{2 T_{E}}
$$

where as explained earlier, the approximation on the right hand side is based on the assumption that the entropy of transformation in independent of temperature (Capdevila et al., 2002; Offerman et al., 2003; Ridley, 1984). We have avoided this by calculating both the enthalpy and entropy changes using MTDATA (NPL, 2006), Fig. 6. Values of $S_{c}$ can be calculated using measured interlamellar spacings from Brown and Ridley (1966) and the entropy production calculations illustrated in Fig. 5a. The interfacial energy derived in this way is illustrated as a function of temperature in Fig. 7. The interfacial energy values calculated here vary from those of Hashiguchi and Kirkaldy (1984), though the spacing and velocity data are in both cases from the same experimental measurements (Brown and Ridley, 1969). This difference may be attributed to two factors:

(i) The $\sigma$ calculated by Hashiguchi and Kirkaldy (1984) is based on the assumption that the entropy change during the pearlite transformation is independent of temperature, whereas we have avoided this approximation.

(ii) The computation of $S_{i} / S_{c}$ based on the maximum entropy production rate was the range 2.18-2.4 (Hashiguchi and Kirkaldy, 1984), whereas in the present 
work it has been shown graphically that this ratio lies in the range of $2.03-$ 2.17 for the temperatures studied. This is attributed to the different growth equations used in the two studies.

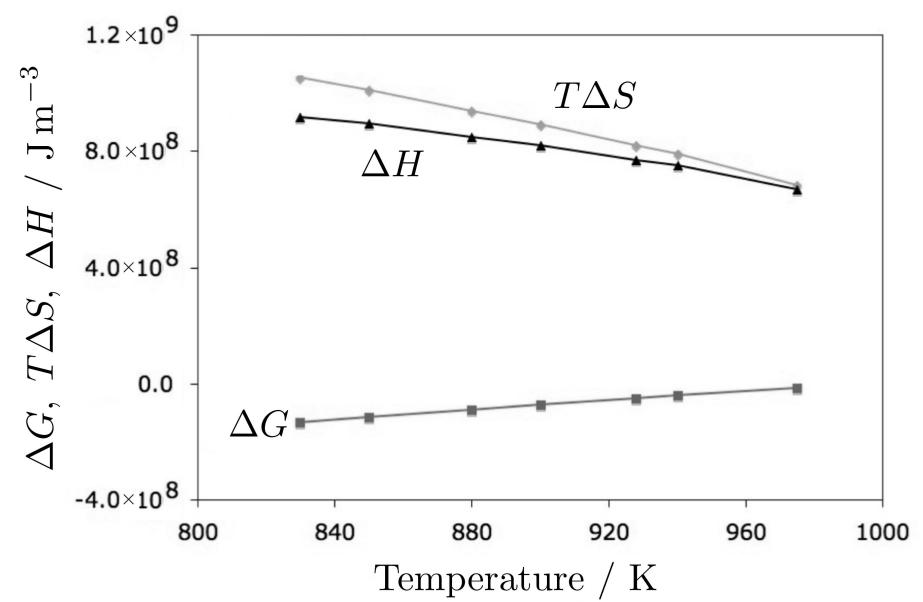

Figure 6. Free energy, enthalpy and entropy change as a function of temperature.

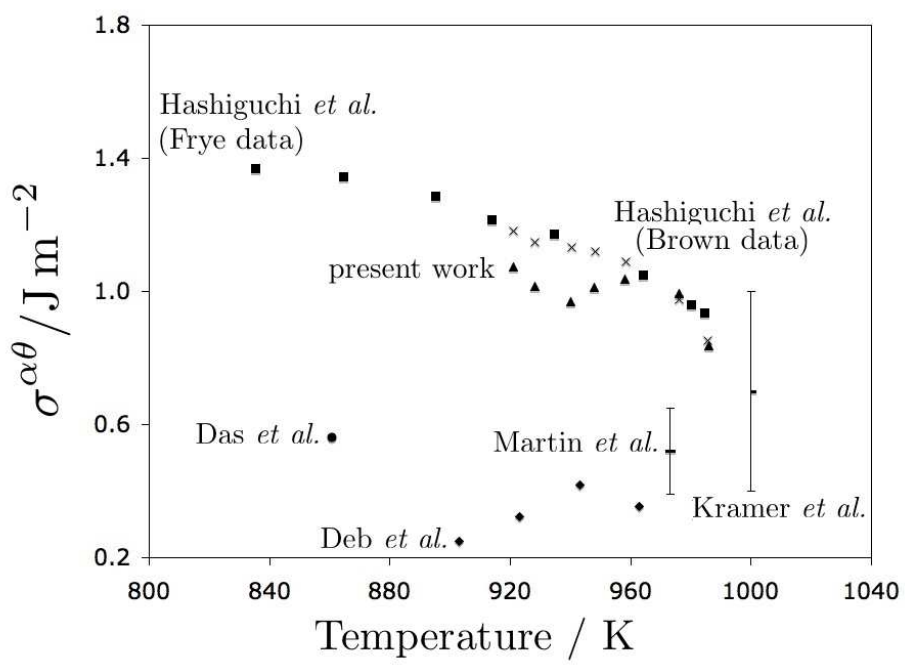

Figure 7. Comparison of calculated ferrite-cementite interfacial energy values and those reported in previous work by Hashiguchi and Kirkaldy (1984).

Independent, published measurements of $\sigma$ are presented in Table 1. Some are estimated on the basis of studies of the coarsening of cementite in ferrite (Das et al., 1993; Deb and Chaturvedi, 1982), from dihedral angle measurements (Martin and Sellars, 1970), calorimetry (Kirchner et al., 1978; Kramer et al., 1958) and simulation (Ruda et al., 2009). Fig. 7 compares the values of interfacial energy derived 
Table 1. Published values of the ferrite-cementite interfacial energy per unit area.

\begin{tabular}{lccl}
\hline reference & temperature $(\mathrm{K})$ & method & $\sigma\left(\mathrm{J} \mathrm{m}^{-2}\right)$ \\
\hline (Das et al., 1993) & 861 & coarsening rate and data fitting & 0.56 \\
(Deb and Chaturvedi, 1982) & $903-963$ & coarsening rate and data fitting & $0.248-0.417$ \\
(Kramer et al., 1958) & 1000 & interfacial enthalpy measurement & $0.7 \pm 0.3$ \\
(Martin and Sellars, 1970) & 973 & dihedral angle & $0.52 \pm 0.13$ \\
(Ruda et al., 2009) & - & atomistic simulation & 0.615 \\
(Kirchner et al., 1978) & - & interfacial enthalpy measurement & $0.5 \pm 0.36$ \\
\hline
\end{tabular}

from pearlite growth rate measurements with the independently measured values. The discrepancies are large for the lower transformation temperatures, relative to the data based on coarsening reactions and dihedral angle measurements. These are both techniques which are kinetically slow; it is possible therefore that the measured values are influenced by the segregation of solutes to the interface, which would lead to a reduction in energy. In contrast, the cementite-ferrite interfaces in pearlite are created fresh as a consequence of transformation. Entropy requires that the extent of segregation should be reduced at high temperatures.

One further difficulty is that the diffusivity $D_{B}$ is likely to increase with interfacial energy since a high value of the latter implies a less coherent interface. We are not able to account for this effect given the absence of relevant grain boundary diffusion data.

Fig. 8 shows a comparison of pearlite growth rates calculated using the theory developed in this paper, against published experimental data due to (Brown and Ridley, 1969; Frye et al., 1953). There is a significant improvement, by a factor of between 2-2.5, over the growth rates calculated by Puls and Kirkaldy (1972).

\section{Conclusions}

A simplified theory has been proposed which combines the contributions from volume and boundary diffusivities, to represent the pearlite growth mechanism in $\mathrm{Fe}-\mathrm{C}$ steels. The match with experimental data is better when compared with prior work, in spite of the fact that considerations of equilibrium at junctions between interfaces are abandoned. As might be expected, the flux through the boundary between pearlite and austenite dominates the transport of carbon at all but the highest of transformation temperatures. The theory for the first time leads to a realistic value for the activation energy for the grain boundary diffusion of carbon, less than that for volume diffusion in austenite and greater than for volume diffusion in ferrite.

The maximum entropy and growth rate criteria have been derived in the context of this mixed mode diffusion theory, with the result that $S_{i} / S_{c}$ is not constant but becomes a function of the transformation temperature. The ferrite-cementite interfacial energy has been deduced assuming that the pearlite interlamellar spacing is determined by the need to maximise the entropy production rate. The energy is lower than determined in previous work, but still much higher than reported in independent experiments, possibly because the interfaces created during transformation are fresh. 


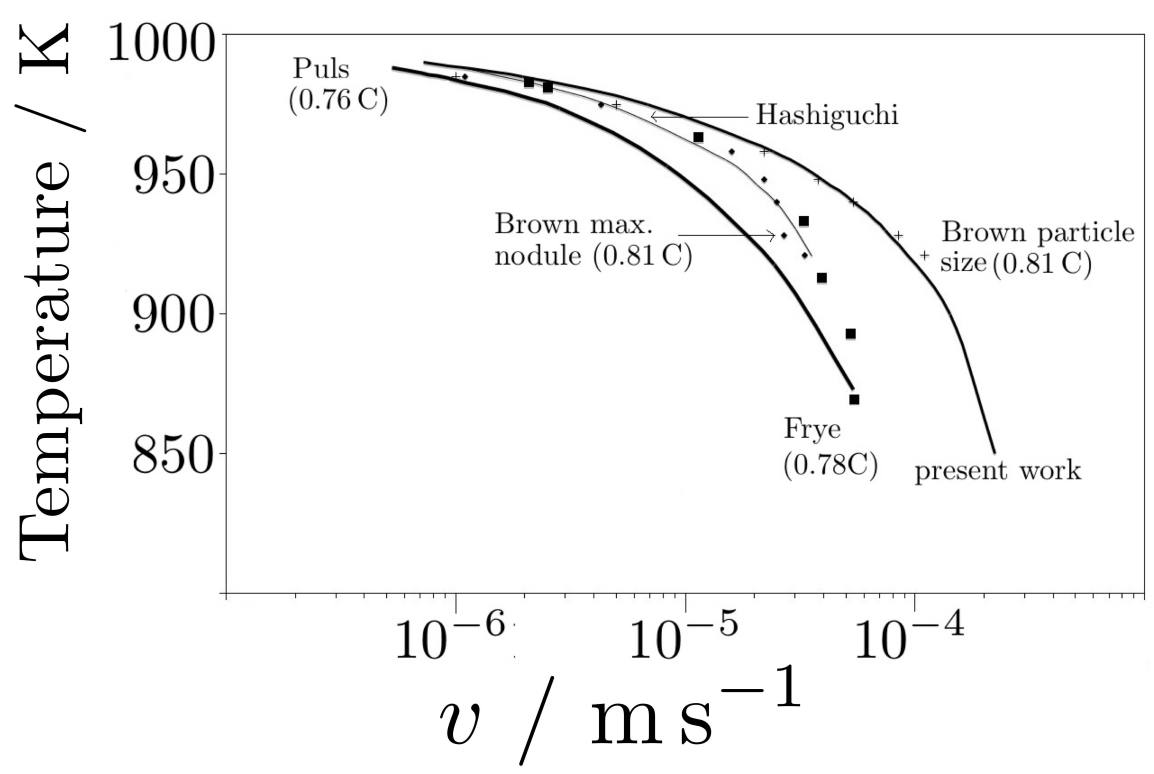

Figure 8. Temperature versus pearlite growth rate plot for Fe-0.8C wt\% steel. Solid lines are calculated. The data from Brown and Ridley (1969) based on particle size analysis are regarded as the most reliable for reasons discussed in the text.

In is argued that this simplified theory avoids many of the approximations required to implement a more complex model in which the shape of the transformation front is determined by equilibrium at interfacial junctions.

\section{Appendix: diffusion in ferrite}

As pointed our previously, Nakajima et al. (2006) used a phase field model to treat the possibility that a flux in the ferrite, behind the pearlite/austenite transformation front, also contributes to the growth rate of pearlite. Their model neglected the flux within the transformation front itself. We present here an analytical treatment which considers the contributions of all three fluxes, that through the volume of the austenite, through the transformation front and within the ferrite. The flux within the ferrite is supposed to lead to the thickening of cementite behind the transformation front. Equation 2.8 then becomes:

$$
\frac{v S^{\alpha} S^{\theta}}{S_{i}}\left(c^{\theta}-c^{\alpha}\right)=2 \bar{D}_{V}\left(c^{\gamma \alpha}-c^{\gamma \theta}\right)+\frac{12 D_{B} \delta\left(c^{\gamma \alpha}-c^{\gamma \theta}\right)}{S_{i}}-\frac{2 D_{\alpha}\left(c_{i}^{\alpha \gamma}-c_{i}^{\alpha \theta}\right)}{x}
$$

where the third term on the right represents the flux within the ferrite, towards the cementite, in a region behind the transformation front. $c_{i}^{\alpha \gamma}$ and $c_{i}^{\alpha \theta}$ represent the concentrations at the respective interfaces, which are not necessarily given by equilibrium since as will be shown below, the thickening of the cementite is interface-controlled, not diffusion-controlled. The average diffusion distance from the ferrite at its interface with austenite, to its interface with cementite is written 
$0.5 x S^{\alpha}$, with $x$ assumed here to be given by the ratio $v / v^{\theta}$; this is because a relatively large pearlite growth rate would lead to greater diffusion distances within the ferrite. The sign of this flux is different from the other two terms because it occurs in the product phase leading to a net reduction in the other two fluxes.

The diffusion coefficient of carbon in ferrite is known accurately (McLellan et al., 1965). The diffusivity is very large and we have calculated that the cementite growth cannot be controlled by the diffusion of carbon, but rather by interfacial mobility. Since interface-controlled growth in this case is much smaller, by three orders of magnitude, the gradient $\Delta c^{\alpha}$ of concentration within the ferrite will be much smaller than by using the equilibrium concentrations $c^{\alpha \gamma}$ and $c^{\alpha \theta}$.

To deal with this, the thickening rate $v^{\theta}$ of cementite is first calculated on the basis of the $\theta / \alpha$ interface mobility $M^{\theta \alpha}$ as follows:

$$
v^{\theta}=M^{\theta \alpha} \Delta G^{\theta \alpha} / V_{m}
$$

where the driving force for cementite growth was calculated using MTDATA NPL (2006). The uncertain value of mobility was taken to be $5 \times 10^{-15} \mathrm{~m}^{4} \mathrm{~J} \mathrm{~s}^{-1}$ from Nakajima et al. (2006); it should strictly be a function of temperature but was assumed constant in the absence of appropriate data.

Using the mobility equation 5.2, the actual gradient within the ferrite is given by a mass balance, that the flux must equal the rate at which the cementite absorbs carbon as it grows:

$$
\frac{D_{\alpha}\left(c_{i}^{\alpha \gamma}-c_{i}^{\alpha \theta}\right)}{0.5 x S^{\alpha}}=v^{\theta}\left(c^{\theta \alpha}-c_{i}^{\alpha \theta}\right)
$$

Fig. 9 shows the results of the three flux model against that involving only boundary and volume diffusion in the austenite. They indicate that inclusion of the flux through the ferrite would indeed lead to an increase in the growth rate, but the model without the flux within the ferrite actually represents the experimental data rather well (Fig. 8). The greatest uncertainty in the three-flux model arises in the mobility of the cementite-ferrite interface for which there are no experimental data. It was also pointed out earlier that evidence for the thickening of cementite behind the transformation front is weak. To summarise, it does not at the moment seem necessary or justified to include any flux within the ferrite to explain pearlite growth data.

\section{Acknowledgments}

The authors value the considerable support of Tata Steel towards this work, and are grateful to Professor Lindsay Greer for the provision of laboratory facilities at the University of Cambridge.

\section{References}

Bhadeshia, H. K. D. H., 1980. Thermodynamics of steels - carbon-carbon interaction energy. Metal Science 14, 230-232.

Bhadeshia, H. K. D. H., 1981. Diffusion of carbon in austenite. Metal Science 15, $477-479$. 


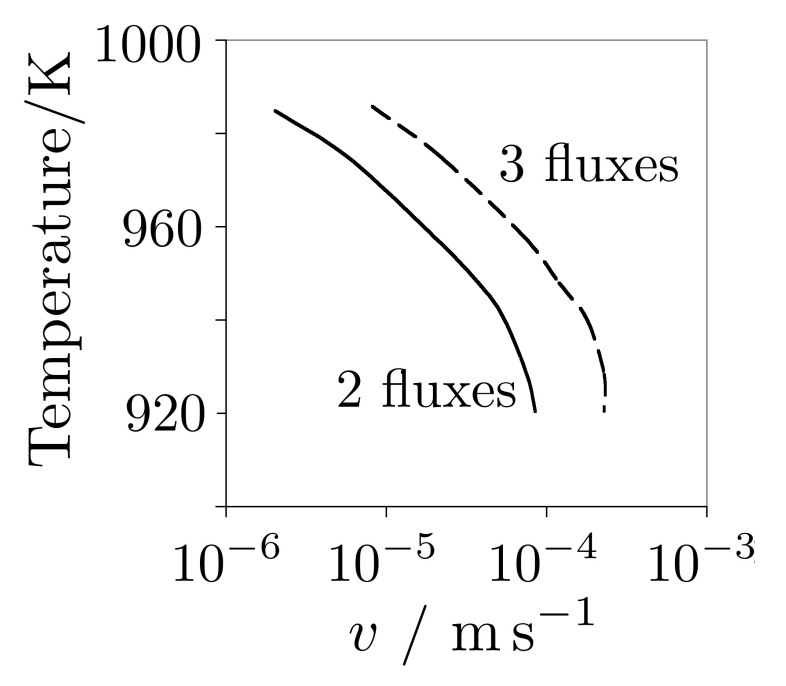

Figure 9. Calculated growth rate of ferrite using the two-flux (volume and interface) and three-flux (volume, interface and through ferrite) models.

Bhadeshia, H. K. D. H., 1985. Diffusional formation of ferrite in iron and its alloys. Progress in Materials Science 29, 321-386.

Brown, D., Ridley, N., 1966. Rates of nucleation and growth and interlamellar spacings of pearlite in a low-alloy eutectoid steel. Journal of the Iron and Steel Institute 204, 811-816.

Brown, D., Ridley, N., 1969. Kinetics of the pearlite reaction in high-purity nickel eutectoid steels. Journal of the Iron and Steel Institute 207, 1232-1240.

Cahn, J. W., Hagel, W. C., 1962. Theory of pearlite reaction. In: Zackay, V. F., Aaronson, H. I. (Eds.), Decomposition of Austenite by Diffusional Processes. Intersciences, New York, pp. 131-192.

Capdevila, C., Caballero, F. G., de Andres, C. G., 2002. Kinetics model of isothermal pearlite formation in a $0.4 \mathrm{C}-1.6 \mathrm{Mn}$ steel. Acta Materialia 50, 4629-4641.

Christian, J. W., 2003a. Theory of Transformations in Metal and Alloys, Part I, 3rd Edition. Pergamon Press, Oxford, U. K.

Christian, J. W., 2003b. Theory of Transformations in Metal and Alloys, Part II, 3rd Edition. Pergamon Press.

Darken, L. S., 1949. Diffusion of carbon in austenite with a discontinuity of composition. Trans. AIME 180, 430-438.

Darken, L. S., Fisher, R. M., 1962. Some observations on the growth of pearlite. In: Zackay, V. F., Aaronson, H. I. (Eds.), Decomposition of austenite by diffusional processes, Interscience publishers. pp. 249-294.

Das, S. K., Biswas, A., Ghosh, R. N., 1993. Volume fraction dependent particle coarsening in plain carbon steel. Acta Metallurgica and Materialia 41, 777-781.

Proc. Royal Society A, 467 (2011) 508-521 
Deb, P., Chaturvedi, M. C., 1982. Coarsening behavior of cementite particles in a ferrite matrix in 10B30 steel. Metallography 15, 341-354.

Dunn, W. W., McLellan, R. B., 1970. The application of a quasichemical solid solution model to carbon in austenite. Metallurgical Transactions 1, 1263-1265.

Frye, J. H., Jr., Stansbury, E. E., McElroy, D. L., 1953. Absolute rate theory applied to rate of growth of pearlite. Trans. A.I.M.E. 197, 219-224.

Graef, M. D., Kral, M. V., Hillert, M., 2006. A modern 3-d view of an old pearlite colony. Journal of Metals 58, 25-28.

Hashiguchi, K., Kirkaldy, J. S., 1984. Pearlite growth by combined volume and phase boundary diffusion. Scandinavian Journal of Metallurgy 13, 240-248.

Hillert, M., 1957. Role of interfacial energy during solid-state phase transformations. Jernkontorets Annaler 141, 757-789.

Hillert, M., 1962. The formation of pearlite. In: Zackay, V. F., Aaronson, H. I. (Eds.), Decomposition of austenite by diffusional processes. Interscience, New York, USA, pp. 197-237.

Hillert, M., 1970. The role of interfaces in phase transformations. In: Mechanism of Phase Transformations in Crystalline Solids. Monograph and Report Series No. 33. Institute of Metals, London, U.K., pp. 231-247.

Hillert, M., 1972. On theories of growth during discontinuous precipitation. Metallurgical Transactions 3, 2729-2741.

Honeycombe, R. W. K., Bhadeshia, H. K. D. H., 1995. Steels: Microstructure and Properties, 2nd edition. Butterworths-Hienemann, London.

Kirchner, H. O. K., Mellor, B. G., Chadwick, G. A., 1978. A calorimetric determination of the interfacial enthalpy of $\mathrm{Cu}-\mathrm{In}$ and $\mathrm{Cu}-\mathrm{Al}$ lamellar eutectoids. Acta Metallurgica 26, 1023-1031.

Kirkaldy, J. S., Sharma, R. C., 1980. Stability principles for lamellar eutectoid(ic) reactions. Acta Metallurgica 28, 1009-1021.

Kral, M. V., Mangan, M. A., Spanos, G., Rosenberg, R. O., 2000. Threedimensional analysis of microstructures. Materials Characterisation 45, 17-23.

Kramer, J. J., Pound, G. M., Mehl, R. F., 1958. The free energy of formation and the interfacial enthalpy in pearlite. Acta Metallurgica 6, 763-771.

Martin, A. G., Sellars, C. M., 1970. Measurement of interfacial energy from extraction replicas of particles on grain boundaries. Metallography 3, 259-273.

McLellan, R. B., Rudee, M. L., Ishibachi, T., 1965. The thermodynamics of dilute interstitial solid solutions with dual-site occupancy and its application to the diffusion of carbon in $\alpha$-iron. Trans. A.I.M.E. 233, 1939-1943.

Proc. Royal Society A, 467 (2011) 508-521 
Nakajima, K., Apel, M., Steinbach, I., 2006. The role of carbon diffusion in ferrite on the kinetics of cooperative growth of pearlite: A multi-phase field study. Acta Materialia 54, 3665-3672.

NPL, 2006. MTDATA. Software, National Physical Laboratory, Teddington, U.K.

Offerman, S. E., van Wilderen, L. J. G. W., van Dijk, N. H., Sietsma, J., th.Rekveldt, M., van der Zwaag, S., 2003. In situ study of pearlite nucleation and growth during isothermal austenite decomposition in nearly eutectoid steel. Acta Materialia 51, 3927-3938.

Pearson, D. D., Verhoeven, J. D., 1984. Forced velocity pearlite in high purity fe-c alloys: Part 1. experimental. Metallurgical Transactions A 15, 1037-1045.

Puls, M. P., Kirkaldy, J. S., 1972. The pearlite reaction. Metallurgical Transactions 3, 2777-2796.

Ridley, N., 1984. The pearlite reaction. In: Marder, A. R., Goldstein, J. I. (Eds.), Phase Transformations in Ferrous Alloys. TMS-AIME, Warrendale, Pennsylvania, USA, pp. 201-236.

Ruda, M., Farkas, D., Garcia, G., 2009. Atomistic simulations in the Fe-C system. Computational Materials Science 45, 550-560.

Siller, R. H., McLellan, R. B., 1970. Application of first order mixing statistics to the variation of the diffusivity of carbon in austenite. Metallurgical Transactions $1,985-988$.

Sundquist, B. E., 1968. The edgewise growth of pearlite. Acta Metallurgica 16, $1413-1427$.

Suwa, Y., Saito, Y., 1997. Two-dimensional grain growth with isotropic grain boundary energy, http://www.msm.cam.ac.uk/phasetrans/abstracts/grain.movies.html.

URL http://www.msm.cam.ac.uk/phase-trans/abstracts/grain.movies.html

Trivedi, R., Pound, G. M., 1967. Effect of concentration dependent diffusion coefficient on the migration of interphase boundaries. Journal of Applied Physics 38, 3569-3576.

Turnbull, D., 1955. Theory of cellular precipitation. Acta Metallurgica 3, 55-63.

Wells, C., Batz, W., Mehl, R. F., 1950. Diffusion coefficient of carbon in austenite. Trans. AIME Journal of Metals 188, 553-560.

Zener, C., 1946. Kinetics of the decomposition of austenite. Trans. Am. Inst. Min. Metall. Engng. 167, 550-595. 